(C) 2020 IEEE. Personal use of this material is permitted. Permission from IEEE must be obtained for all other uses, in any current or future media, including reprinting/republishing this material for advertising or promotional purposes, creating new collective works, for resale or redistribution to servers or lists, or reuse of any copyrighted component of this work in other work.

\title{
Rheumatoid Arthritis miRNA biomarker detection by means of LMR based fiber-optic biosensor
}

\author{
J. J. Imas*1, C. R. Zamarreño ${ }^{1,2}$, P. Zubiate ${ }^{1}$, J. Campión ${ }^{3}$, L. Sánchez-Martín ${ }^{3}$, I. R. Matías ${ }^{1,2}$ \\ ${ }^{1}$ Electrical, Electronic and Communications Engineering Department, Public University of Navarra, 31006 Pamplona, Spain \\ ${ }^{2}$ Institute of Smart Cities (ISC), Public University of Navarra, 31006 Pamplona, Spain \\ ${ }^{3}$ Making Genetics S.L., Plaza CEIN 5, 31110 Noáin, Spain \\ *josejavier.imas@unavarra.es
}

\begin{abstract}
Development of miRNA optical biosensors for disease diagnosis and monitoring has acquired relevance in recent years, due to the clinical importance of miRNA and the inherent advantages of optical sensors. Here, we present the utilization of a fiber optic sensor based on Lossy Mode Resonance (LMR) for the detection of miRNA hsa-miR-223, a promising biomarker for the diagnosis of rheumatoid arthritis (RA).
\end{abstract}

Keywords-miRNA, optical biosensor, Lossy Mode Resonance (LMR), rheumatoid arthritis (RA)

\section{INTRODUCTION}

MicroRNAs or miRNAs are small (around 20 nucleotides in length) and non-coding RNAs (ribonucleic acids) involved in the regulation of gene expression. In recent years, the implication of miRNAs in human diseases has been thoroughly studied, including autoimmune diseases such as rheumatoid arthritis (RA) [1]-[5].

Rheumatoid arthritis (RA) is the most common type of autoimmune and inflammatory arthritis in adults, affecting around $0.5-1.0 \%$ of the adult population worldwide [6]. In rheumatoid arthritis, inflammation is misdirected to attack the synovium, which is the membrane that lines the joint. The inflammation causes joint pain, stiffness, swelling and loss of function, and if it is not stopped or slowed, it can permanently damage the affected joints as well as other tissues (ligaments, tendons, nerves, blood vessels) [7].

Among the miRNAs that play a role in RA, it can be found hsa-miR-223-3p, also known as hsa-miR-223 [8]. For the rest of this paper, the name 'miR-223' will be used, a common abbreviation in literature when talking about miRNAs ('hsa' means the miRNA corresponds to the species Homo Sapiens). Low serum levels of miR-223 compared with both healthy individuals and established RA patients have been associated with early rheumatoid arthritis (ERA) [4]. In the case of patients with established RA, increased serum miR-223 levels are considered to be connected with higher disease activity and disease relapse [4] and a correlation has been found in $\mathrm{T}$ cells between miR-223 and rheumatoid factor (RF), another biomarker for RA [9]. Finally, miR-223 is also believed to be a suitable marker for studying therapeutic response in RA in case of some drugs (DMARDs, anti-TNF $\alpha$ ) [4], [10], that is, to determine if the treatment is effective or not. Nevertheless, the mechanisms

This research was funded by the Spanish Agencia Estatal de Investigación (AEI) (PID2019-106231RB-I00), the Public University of Navarra (PJUPNA26), and the Spanish Ministry of Universities (FPU18/03087). In addition, this project has received funding from the ATTRACT call financed by the European Union's Horizon 2020 research and innovation program under grant agreement No 777222 . that lie under the connection between miR-223 and RA are complex and need further research [1]-[5], [10], [11], so here the explanation has been synthesized for the sake of simplicity.

On the other hand, fiber optical biosensors are an emerging technology that advances towards reliable, highly sensitive, reusable, and fully automated platforms for the detection of biomarkers [12]. Among them, sensors based on Lossy Mode Resonances (LMR) have emerged as a high sensitivity refractometric platform [13], [14]; which enables their use for biosensing applications [15], [16]. LMR occurs when the real part of the thin-film permittivity is positive and greater in absolute value than its own imaginary part and the real part of the permittivity of the waveguide, so metallic oxides and polymers can be employed to generate these resonances [17].

In this work, it is presented the fabrication of LMR-based fiber optic refractometers with $\mathrm{SnO}_{2}$ thin-films on D-shaped optical fibers, and their utilization as biosensors for the detection of miR-223, a biomarker associated to rheumatoid arthritis.

\section{METHODS AND MATERIALS}

\section{A. Refractometer manufacture}

LMR-based optical fiber refractometers have been described in previous works [18]. Here, D-shaped singlemode fiber (SMF) purchased from Phoenix Photonics Ltd., is employed as substrate. D-shape fiber consists of a standard SMF (SMF-28, Corning ${ }^{\circledR}$ ) with a side-polished length of 10 $\mathrm{mm}$ and an attenuation peak of $0.5 \mathrm{~dB}$ at $1550 \mathrm{~nm}$ in high refractive index oil (1.5).

A thin-film of $\mathrm{SnO}_{2}$ was deposited onto the D-shape region to generate the LMR. Regarding the deposition procedure, the fiber is placed inside a DC sputtering machine (K675XD from Quorum Technologies) at a partial pressure of argon of $9 \times 10^{-2}$ mbar and an intensity of $90 \mathrm{~mA}$ in order to achieve $100 \mathrm{~nm}$ thick $\mathrm{SnO}_{2}$ coatings. Subsequently, this structure is biofunctionalized to allow the detection of miR223, as it is described in subsection B (see Fig. 1a, b).

\section{B. Surface functionalization and bioassay protocol}

Once the D-shaped SMF fiber has been coated with a $\mathrm{SnO}_{2}$ thin-film, there are three different parts that can be distinguished in the biosensor manufacture and operation: 1) initial biofunctionalization steps, 2) DNA probe (the bioreceptor) binding, and 3) miRNA (the biomarker, that is, 

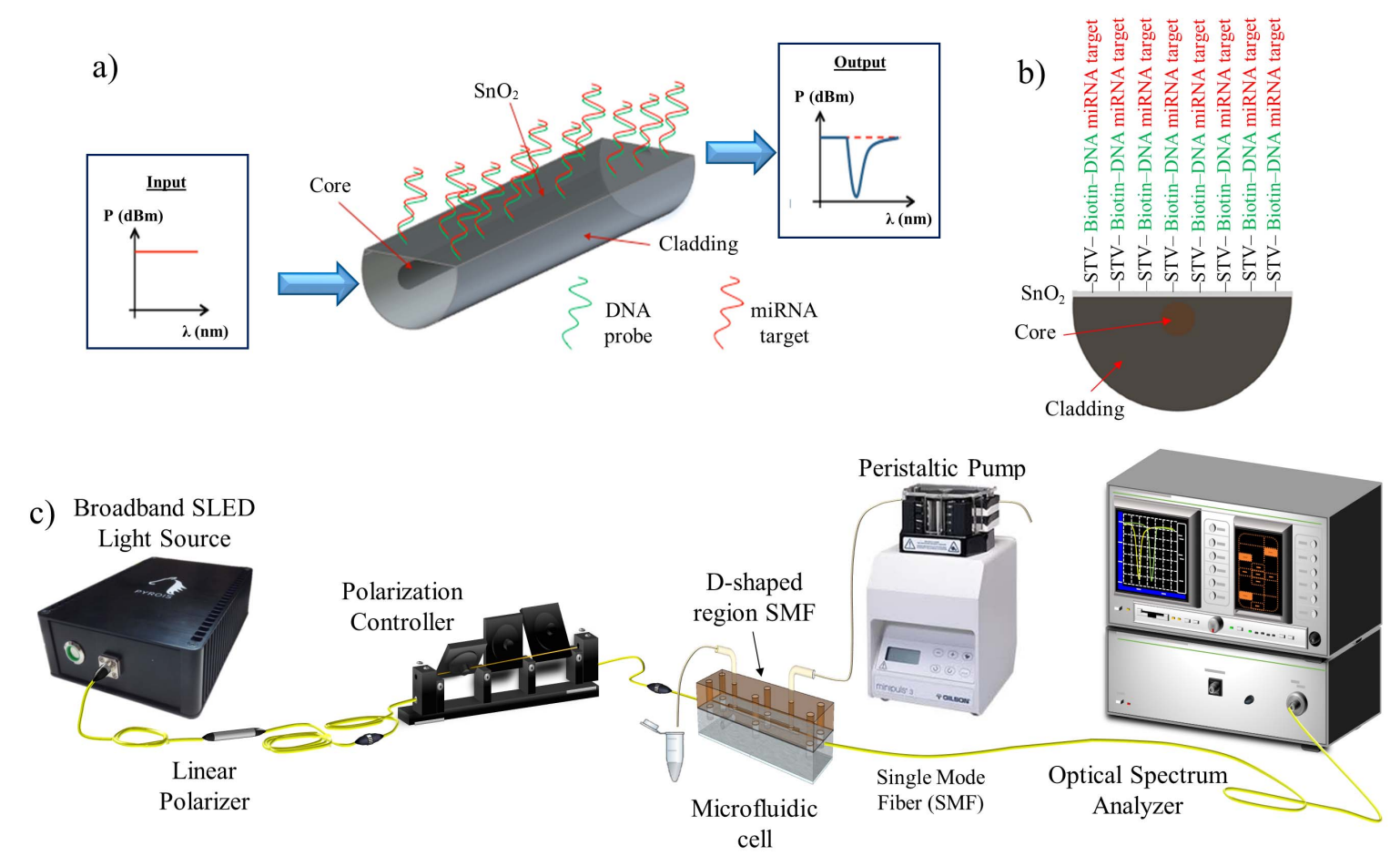

Fig. 1. a) Schematic representation of the optical fiber biosensor based on Lossy Mode Resonance. b) Schematic representation of the biofunctionalization and sensing principle of the biosensor. c) Experimental setup scheme.

miR-223) detection. All the binding interactions were performed in situ and were continuously monitored. More detailed information is given in section III.

\section{Measuring setup}

The experimental setup is detailed in Fig. 1c, consisting of a broadband multi-SLED light source (FJORD-X2-13301550 purchased from Pyroistech S.L.), an in-line polarizer, and a polarization controller (purchased from Phoenix Photonics Ltd.), which enable selecting TE- or TM-polarized states of light. An optical spectrum analyzer (MS9740A, purchased from Anritsu), is connected to the output to monitor the biosensor response in the 1200-1700 nm range.

\section{RESULTS AND DISCUSSION}

\section{1) Initial biofunctionalization steps}

In the first place, the D-shaped region of the SMF fiber is immersed in a $0.04 \% \mathrm{w} / \mathrm{v}$ solution of Eudragit ${ }^{\circledR}$ in $99 \%$ pure alcohol for $1 \mathrm{~min}$ and then it is left drying in air for about 15 min until the solvent has completely evaporated.

After this step, the D-shaped region is placed inside a microfluidic system that is thermo-stabilized by means of two Peltier cells. The microfluidic system consists of two equal-size pieces, the upper one is made of ULTEM ${ }^{\circledR}$ and the lower one is made of stainless steel. A microfluidic channel is engraved on both bars with dimensions $1 \mathrm{~mm} \times 1$ $\mathrm{mm} \times 50 \mathrm{~mm}$ (volume of $50 \mu \mathrm{L}$ ), with the D-shaped region fitting in this channel. A peristaltic pump allows to control the flow through the cell.

Then, once the D-shaped region is correctly placed, a 1Ethyl-3-(3-dimethylaminopropyl) carbodiimide hydrochlo- ride (EDC) and N-Hydroxysuccinimide (NHS) solution (2 $\mathrm{mM}$ and $5 \mathrm{mM}$ in ultrapure water, $18.1 \mathrm{M} \Omega / \mathrm{cm}$ ) is injected and flowed through the cell during $30 \mathrm{~min}$ at a flow rate of $4.4 \mu \mathrm{L} / \mathrm{min}$.

\section{2) DNA probe binding}

In order to detect the target miRNA, a first binding of a DNA probe to the D-shaped fiber is required. The DNA probe is complementary to the target miRNA (miR-223). This binding induces a change in the thickness of the coating and in the effective refractive index of the outer medium. Therefore, an accurate determination of this change can be obtained by studying the LMR wavelength shift.

The DNA probe is immobilized onto the fiber by employing biotin as linker but, apart from the biofunctionalization operations explained in 1), the following steps are required. In all the cases, the flow rate is 4.4 $\mu \mathrm{L} / \mathrm{min}$. and the buffer that is employed is phosphate buffered saline (10 mM PBS, $\mathrm{pH} 7.4$ ).

a) Streptavidin (STV, 0.03\% w/v) is flowed during $1 \mathrm{~h}$

b) PBS buffer during 15 min (washing step to recover the baseline)

c) Until this point of the bioassay, the temperature of the microfluidic cell has been stabilized at $25^{\circ} \mathrm{C}$. Here, it is changed to $32^{\circ} \mathrm{C}$ to favor the binding between the DNA probe and the miRNA (miR-223), as it has been considered an adequate temperature. It will maintain this value for the rest of the assay.

d) PBS buffer during $15 \mathrm{~min}$ after the temperature has stabilized to its new value (steps $d$-f are shown in Fig. 2). 


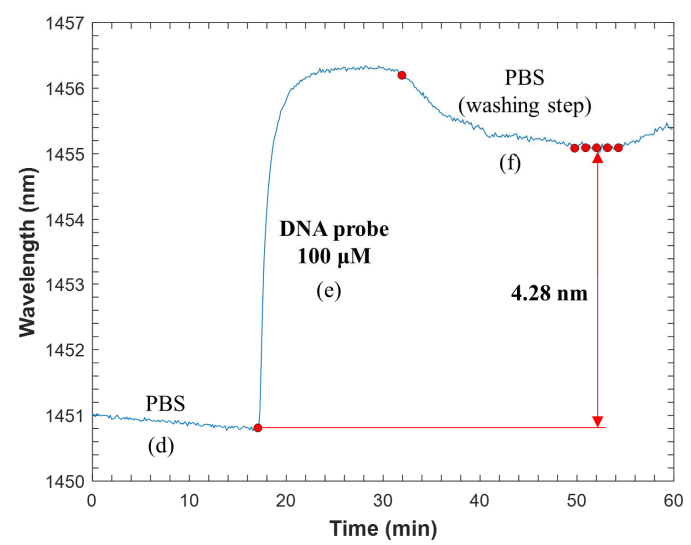

Fig. 2. Sensogram corresponding to the DNA probe binding (steps d-f).

e) DNA probe $(100 \mu M)$ during $15 \mathrm{~min}$. This DNA probe has been preheated at $50^{\circ} \mathrm{C}$ during 10 min to favor its unfolding. The DNA probe is complementary to the miRNA (miR-223) that must be detected, and its sequence is: 5'-Biotin- TGG GGT ATT TGA CAA ACT GAC A-3' (note that it has an additional Biotin linker)

\section{f) PBS buffer during 15 min (washing step).}

Fig. 2 shows the evolution of the central wavelength of the LMR during the steps d) - f), once the temperature change (step c) has been carried out. It can be observed that the LMR wavelength shift associated to the DNA probe binding is $4.28 \mathrm{~nm}$ (initial change minus the change backwards in PBS).

Step c) is done because, ideally, the working temperature must be higher than the melting temperature of both the DNA probe and the miRNA $\left(30.9^{\circ} \mathrm{C}\right.$ and $23.1^{\circ} \mathrm{C}$ respectively, in this case) and lower than the melting temperature of the hybridization, that is, the binding of the DNA probe and the miRNA $\left(70.9^{\circ} \mathrm{C}\right.$ in this case).

This happens because the melting temperature of a hairpin, such as the DNA probe or the miRNA, is the temperature at which the corresponding hairpin can be considered unfolded, that is, a working temperature that is higher than this value favors the binding. On the other hand, the melting temperature of hybridization is the value at which half of the unions among the DNA probe and the target miRNAs are considered to be unlinked, so the working temperature must be lower than this value.

\section{3) miRNA (miR-223) detection}

Finally, it must be studied if the biosensor detects the miRNA target, that is, the miR-223. The sequence of miR223 is: 5'- TGT CAG TTT GTC AAA TAC CCC A-3'. Right now, DNA is being employed in these assays instead of RNA (thymine, T, instead of uracil, $\mathrm{U}$ ). In this part of the assay, the buffer continues being PBS and the flow rate, 4.4 $\mu \mathrm{L} / \mathrm{min}$. The following elements are injected and flow through the cell (please note that step g) follows step f)):

g) Bovine serum albumin (BSA, 0.04\% w/v) for $10 \mathrm{~min}$ for surface passivation..
h) Target miR-223 (0.02 $\mu M)$ for $15 \mathrm{~min}$
i) Target miR-223 (2 $\mu \mathrm{M})$ for $15 \mathrm{~min}$

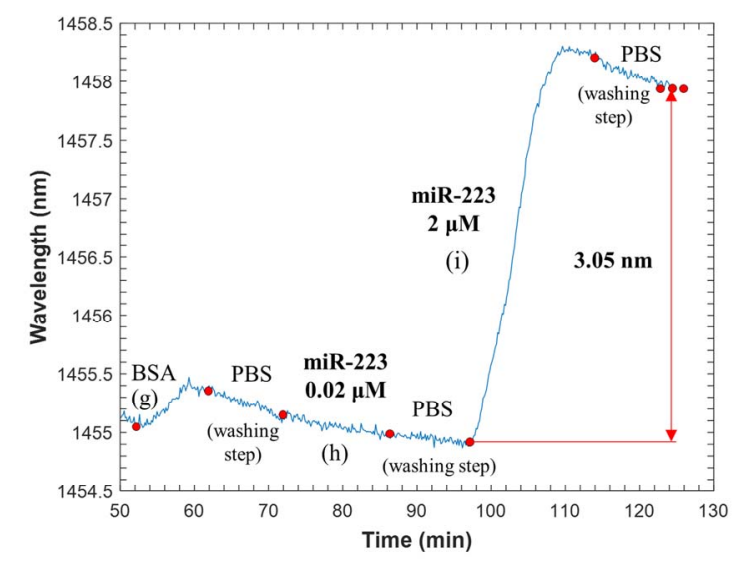

Fig. 3. Sensogram corresponding to the miR-223 detection.

After each of these three steps, a washing step (10 min) is done in PBS. All this process can be observed in Fig. 3. All miRNAs were preheated at $50^{\circ} \mathrm{C}$ for $10 \mathrm{~min}$.

BSA is employed to fill in the spaces between the DNA probes, producing a shift in step g), see Fig. 3. This way, it is ensured that the subsequent LMR shift caused by the target miRNAs is only due to their binding to the DNA probes.

As in the case of the DNA probe, the binding of the miR223 to the DNA probe is detected through the LMR wavelength shift. Nevertheless, as it is shown in Fig. 3, a $0.02 \mu \mathrm{M}$ concentration of target miR-223 does not produce an appreciable LMR wavelength shift, whereas a $2 \mu \mathrm{M}$ concentration of the target causes a shift of $3.1 \mathrm{~nm}$. After the flow of each miR-223 concentration, a PBS washing step is done to determine the effective wavelength shift $(0$ and 3.1 $\mathrm{nm}$ respectively). It is clear the limit of detection of this biosensor, with the current configuration, is between 0.02 and $2 \mu \mathrm{M}$, Further research must be carried out in order to precisely establish this value and whether it can be improved.

\section{CONCLUSIONS}

The detection of a miRNA has been performed in this work by means of a LMR-based fiber optic biosensor. The LMR has been generated by depositing a $\mathrm{SnO}_{2}$ thin-film onto a D-shaped optical fiber. The surface is biofunctionalized by employing streptavidin and biotin, and the binding of the DNA probe and the target miRNA (miR-223) is monitored in real time.

Further research is required to improve the detection limit. The optimal working temperature for the binding of the DNA probe and the target must be studied in depth. The employment of RNA instead of DNA in the case of the target and the utilization of other buffers and biofunctionalization procedures are also other possibilities that need to be considered to lower the detection limit.

Nevertheless, it has been demonstrated that the use of a LMR-based fiber optic biosensor is possible for miRNA detection, which opens great possibilities in this field. All miRNAs are quite similar in length (around 20 nucleotides) and characteristics, so if this biosensor has enabled the detection of miR-223, with small changes (working temperature, use of the corresponding DNA probe) it should be capable of detecting whatever target miRNA. 


\section{REFERENCES}

[1] M. Birlik, A. Koçak, and D. Harmanci, "Role of MicroRNAs in Rheumatoid Arthritis," in New Developments in the Pathogenesis of Rheumatoid Arthritis, InTech, 2017.

[2] M. C. Moran-Moguel, S. P. Del Rio, E. E. Mayorquin-Galvan, and M. G. Zavala-Cerna, "Rheumatoid arthritis and miRNAs: A critical review through a functional view," Journal of Immunology Research, vol. 2018. Hindawi Limited, 2018.

[3] R. Y. Huang, J. Q. Wu, Z. H. Liu, and S. L. Sun, "MicroRNAs in rheumatoid arthritis: what is the latest with regards to diagnostics?," Expert Review of Molecular Diagnostics, vol. 19, no. 5. Taylor and Francis Ltd, pp. 363-366, 04-May-2019.

[4] G. Evangelatos, G. E. Fragoulis, V. Koulouri, and G. I. Lambrou, "MicroRNAs in rheumatoid arthritis: From pathogenesis to clinical impact," Autoimmunity Reviews, vol. 18, no. 11. Elsevier B.V., p. 102391, 01-Nov-2019.

[5] A. V. Churov, E. K. Oleinik, and M. Knip, "MicroRNAs in rheumatoid arthritis: Altered expression and diagnostic potential," Autoimmunity Reviews, vol. 14, no. 11. Elsevier, pp. 1029-1037, 01-Nov-2015.

[6] G. S. Firestein, "Evolving concepts of rheumatoid arthritis," Nature, vol. 423, no. 6937. Nature Publishing Group, pp. 356361, 15-May-2003.

[7] C. B. Driver and M. C. Stöppler, "Rheumatoid Arthritis (RA): Early Signs, Symptoms, Causes, Treatment \& Diet." [Online]. Available:

https://www.emedicinehealth.com/rheumatoid_arthritis/article_e m.htm\#rheumatoid_arthritis_ra_definition_and_facts.

[8] S. Griffiths-Jones et al., "miRNA Entry for MI0000300." [Online]. Available: http://www.mirbase.org/cgibin/mirna_entry.pl?acc=MI0000300. [Accessed: 13-Jul-2020].

[9] M.-C. Lu, C.-L. Yu, H.-C. Chen, H.-C. Yu, H.-B. Huang, and N.S. Lai, "Increased miR-223 expression in T cells from patients with rheumatoid arthritis leads to decreased insulin-like growth factor-1-mediated interleukin-10 production," Clin. Exp. Immunol., vol. 177, no. 3, pp. 641-651, Sep. 2014.

[10] A. Paradowska-Gorycka and B. Stypinska, "MicroRNAs in Rheumatoid Arthritis: From Pathogenesis to Clinical Utility," in New Developments in the Pathogenesis of Rheumatoid Arthritis, InTech, 2017.

[11] K. Murata et al., "Comprehensive microRNA Analysis Identifies miR-24 and miR-125a-5p as Plasma Biomarkers for Rheumatoid Arthritis," PLoS One, vol. 8, no. 7, Jul. 2013.

[12] C. R. Zamarreño et al., "Optical Fibers: Biosensors," in Encyclopedia of Optical and Photonic Engineering, Second Edition, CRC Press, 2019, pp. 1-19.

[13] F. J. Arregui, I. Del Villar, C. R. Zamarreño, P. Zubiate, and I. R. Matias, "Giant sensitivity of optical fiber sensors by means of lossy mode resonance," Sensors Actuators, B Chem., vol. 232, pp. 660-665, Sep. 2016.

[14] A. Ozcariz, C. R. Zamarreño, P. Zubiate, and F. J. Arregui, "Is there a frontier in sensitivity with Lossy mode resonance (LMR) based refractometers?," Sci. Rep., vol. 7, no. 1, pp. 1-7, Dec. 2017.

[15] P. Zubiate, C. R. Zamarreño, P. Sánchez, I. R. Matias, and F. J. Arregui, "High sensitive and selective C-reactive protein detection by means of lossy mode resonance based optical fiber devices," Biosens. Bioelectron., vol. 93, pp. 176-181, Jul. 2017.

[16] P. Zubiate et al., "Fiber-based early diagnosis of venous thromboembolic disease by label-free D-dimer detection," 2019.

[17] I. Del Villar et al., "Optical sensors based on lossy-mode resonances," Sensors and Actuators, B: Chemical, vol. 240. Elsevier B.V., pp. 174-185, 01-Mar-2017.

[18] P. Zubiate, C. R. Zamarreño, I. Del Villar, I. R. Matias, and F. J. Arregui, "High sensitive refractometers based on lossy mode resonances (LMRs) supported by ITO coated D-shaped optical fibers," Opt. Express, vol. 23, no. 6, p. 8045, Mar. 2015. 